\title{
Schistosomiasis, soil transmitted helminthiasis, and malaria co-infections among women of reproductive age in rural communities of Kwale County, coastal Kenya
}

Victor Tunje Jeza ${ }^{1 *}$, Francis Mutuku², Lydia Kaduka ${ }^{3}$, Charles Mwandawiro ${ }^{4}$, Janet Masaku ${ }^{4}$, Collins Okoyo $^{4}$, Henry Kanyi ${ }^{4}$, Joyce Kamau ${ }^{4}$, Zipporah Ng'ang' ${ }^{5}$ and Jimmy Hussein Kihara ${ }^{4}$

\begin{abstract}
Background: Schistosoma haematobium, soil transmitted helminthes (STH), and malaria lead to a double burden in pregnancy that eventually leads to poor immunity, increased susceptibility to other infections, and poor pregnancy outcomes. Many studies have been carried out on pre-school and school aged children but very little has been done among the at risk adult population including women of reproductive age (WRA). Our current study sought to establish the risk factors and burden of co-infection with S. haematobium, STH, and Plasmodium sp. among WRA in Kwale County, Coastal Kenya.
\end{abstract}

Methods: A total of 534 WRA between the ages of 15-50 were enrolled in this cross-sectional study from four villages; Bilashaka and Mwaluphamba in Matuga sub-County, and Mwachinga and Dumbule in Kinango sub-County. Socio-demographic information was collected using a pre-tested standardized questionnaire. Parasitological examination was done using urine filtration method for Schistosoma haematobium, Kato Katz for STH (Ascaris lumbricoides, Hookworm, Trichuris trichiura), and standard slide microscopy for Plasmodium sp. Statistical analyses were carried out using STATA version 15.1.

Results: The overall prevalence of S. haematobium was 3.8\% (95\% Cl: 2.6-5.4) while that for malaria was 4.9\% (95\% Cl: $2.0-11.7)$. The prevalence of STH was 5.6\% (95\% Cl: 2.8-11.3) with overall prevalence of 5.3\% (95\% Cl: $2.5-10.9)$ for hookworm and $0.6 \%$ (95\% Cl: 0.2-1.9) for T. trichiura. The occurrence of co-infection was low and was recorded between S. haematobium and P. falciparum (0.6\%), followed by S. haematobium and STH (0.4\%).

Among pregnant women, $2.6 \%$ had co-infection with S. haematobium and P. falciparum. Only $1.3 \%$ had co-infection with S. haematobium and hookworm or T. trichiura. Among non-pregnant women, co-infection with S. haematobium and P. falciparum was $0.2 \%$. Similarly, co-infection with S. haematobium and hookworm or T. trichiura was $0.2 \%$.

Bed net ownership and usage among pregnant women was 87.8 and $96.6 \%$, respectively. $66.3 \%$ of the women reported using improved water sources for drinking while $78.1 \%$ reported using improved sanitation facilities.

\footnotetext{
*Correspondence: vjeza@tum.ac.ke

1 Department of Medical Sciences, Technical University of Mombasa,

Mombasa, Kenya

Full list of author information is available at the end of the article
}

(C) The Author(s) 2022. Open Access This article is licensed under a Creative Commons Attribution 4.0 International License, which permits use, sharing, adaptation, distribution and reproduction in any medium or format, as long as you give appropriate credit to the original author(s) and the source, provide a link to the Creative Commons licence, and indicate if changes were made. The images or other third party material in this article are included in the article's Creative Commons licence, unless indicated otherwise in a credit line to the material. If material is not included in the article's Creative Commons licence and your intended use is not permitted by statutory regulation or exceeds the permitted use, you will need to obtain permission directly from the copyright holder. To view a copy of this licence, visit http://creativecommons.org/licenses/by/4.0/. The Creative Commons Public Domain Dedication waiver (http://creativeco mmons.org/publicdomain/zero/1.0/) applies to the data made available in this article, unless otherwise stated in a credit line to the data. 
Conclusion: The use of improved WASH activities might have contributed to the low prevalence of STHs and S. haematobium infections. Further, bed net ownership and usage might have resulted in the low prevalence of Plasmodium sp. infections observed.

Keywords: S. Haematobium, STH, Malaria, Co-infections, WRA, Coastal Kenya

\section{Background}

Schistosomiasis is one of the neglected tropical diseases (NTDs) caused by trematodes of the genus Schistosoma that infects a human host after coming in contact with water infested with snails habouring human schistosome cercariae. Three major species of the genus Schistosoma are major contributors to schistosomiasis in humans around the world, viz.; Schistosoma mansoni, Schistosoma japonicum, and Schistosoma haematobium [1]. Schistosomiasis causes morbidities and mortalities with an estimated 230 million people affected worldwide [2], making it one of the NTDs causing huge socio-economic and public health concerns. The most affected are the poor in developing countries especially in sub-Saharan Africa. Poverty is both cause and effect of the disease burden, consequently, schistosomiasis survives in poverty stricken areas, that are remote with little or no safe water and sanitation, and scarce or non-existence of health care facilities [3]. Schistosomiasis affects people across all age groups and gender. However, it affects children and women more than men and the effects of infection tend to be greater in women of reproductive age (WRA) and especially pregnant women $[3,4]$. It has been shown that worms, which include $S$. haematobium and soil transmitted helminthes $(\mathrm{STH})$, and malaria lead to a double burden in pregnancy that eventually leads to reduction in immunity, increased susceptibility to other infections, and poor pregnancy outcomes such as low birth weight and other developmental problems [4-6].

Approximately 40 million women of reproductive age are infected with S. haematobium, S. japonicum, and/or S. mansoni around the world [7, 8]. In Kenya, an estimated 6 million people are infected with schistosomiasis [9], with the majority being women and children, while another approximately 10 million people are infected with STH [10]. The prevalence of S. haematobium in Kwale (similar study site to the current study) among adults was $18.2 \%$ in 2010 [11] and $15.07 \%$ in 2018 among people of all age groups and gender [12]. Three major STH infections are caused by roundworms (Ascaris lumbricoides), whipworms (Trichuris trichiura), and the hookworms (Ancylostoma duodenale and/or Necator americanus) [13-15]. Generally a combination of factors have been implicated in STH infections which include poverty, lack of sanitation, and inadequate hygiene [15-17]. Consequently, combating these factors tends to reduce the infections. It has also been shown that school based deworming (SBD) reduces the burden of STH infections. However, Anderson et al [18] have shown that SBD alone may not be very effective at reducing or interrupting transmission at the community level. A number of studies have shown that SBD in combination with community based deworming (CBD) where mass drug administration (MDA) to the community is done is the key to effectively control STH infections $[19,20]$. The Tuangamize Minyoo Kenya Imarisha Afya (TUMIKIA) project, a cluster randomized trial done in Kwale County to determine the effectiveness of SBD and CBD also concurs with the fact that MDA at the community level is more effective in reducing the prevalence and intensity of STH infection [20-25]. Notably, most STH infections in the study area occur in young children with low prevalence recorded in the adult population. In a study in Kwale published in 2015, Kihara et al [26] found a prevalence of $2.6 \%$ among women of reproductive age.

Malaria (Plasmodium sp.) infection, which is also transmitted in areas where schistosomiasis occurs, has been diagnosed together with schistosomes. Although not a NTD, malaria is a common infection in Africa and of public health concern. It affects the poor and also contributes to poverty through lost savings, lost man hours, reduction of income, expenses for preventive measures, and deaths, leading to reduction in human resource. Malaria caused an estimated 229 million clinical cases and an estimated 409,000 deaths in 2019 [27] with an estimated $90 \%$ of those deaths occuring in sub-Saharan Africa [27]. The most affected are young children who have not fully developed their protective immunity and pregnant women whose immunity to malaria is downregulated. The prevalence of malaria was recorded as 0 $\%$ among women of reproductive age in 2015 in Kwale [26] and 6\% nationally among children aged 6 months to 14 years in 2020 [28].

A significant number of studies have been done in Africa in general and Kenya in particular on schistosomiasis, STH, and malaria. The coastal region of Kenya has particularly received a fair share of these studies on schistosomiasis due to its endemicity in the region [29]. Most of the studies have however, concentrated on pre-school and school aged children [30-39] and very little has been done among the adult population including WRA. Our current study aimed at understanding 
the risk factors and burden of co-infection with $S$. haematobium, STH, and Plasmodium sp. among WRA in Kwale County, coastal region of Kenya.

\section{Methods \\ Study design and study site}

This cross-sectional study was carried out in November of 2018 in Kwale County, South Coast, Kenya. The County borders Taita Taveta County to the West, Kilifi County to the Northwest, Mombasa County to the North, the Indian Ocean to the East, and the Republic of Tanzania to the South [40]. The county is located in the Southeastern corner of Kenya, at Latitudes $3^{\circ} 3$ "and $4^{\circ} 45^{\prime}$ South and longitudes $38^{\circ} 31^{\prime}$ and $39^{\circ} 31^{\prime}$ East [40]. Kwale County is divided into 5 administrative subcounties; Kinango, Lunga Lunga, Matuga, Msambweni, and Samburu according to the 2019 Kenya Population and Housing Census [41]. Kwale is usually hot and dry from January to March. Monsoonal heavy rains begin in mid-March and last up to June. The months of June, July, August, and September are usually cool with July being the coldest. Short rains start in October and end in early December [42]. Majority of the population practice subsistence farming of crops such as maize and cassava. A few cash crops are farmed which include coconuts, oranges, and mangoes. The area is dotted with dams and seasonal streams [26].

\section{Study population and sample size}

According to the 2019 Kenya Population and Housing Census, Kwale County had a total population of 858,748 by 2019 [41]. The sample size of WRA was calculated using the Cochran formula [43]; $n=Z^{2} p q / e^{2}$; where $\mathrm{Z}$ is the score for a $5 \%$ type 1 error for a normal distribution $(Z=1.96), p$ is the prevalence of urogenital schistosomiasis in WRA taken as 36\% [26]. This prevalence $(36 \%)$ was used in this study because of the similarity of that particular study by Kihara et al [26] to our current study in terms of the infection, study population, and the study site which is one of the villages where we collected data and samples from. The proportion of the population with no infection is represented by the letter q. Using a margin of error (e) of 4.5 and $19 \%$ non-response rate, the final estimated sample size was 534 WRA. Hence, a total of 534 WRA between the ages of 15-50 were enrolled in this cross-sectional study from four randomly selected villages of Bilashaka, Mwaluphamba, Mwachinga, and Dumbule (Fig. 1). The four villages under study were in Matuga and Kinango sub-counties with a population of 192,999 and 93,789 respectively [41].

\section{Community mobilization and sensitization}

Before commencement of the study, meetings were held with the county, sub-county, location, sub-location, village leaders, and the community to sensitize them about the study. During this sensitization period, the study participants were requested to visit the nearest health facility in the area on specific dates for sample and data collection. Two villages that were served by a health facility were randomly selected in each sub-county. The selected health facilities served as sample collection centers. They included Bilashaka and Mwaluphamba in Matuga sub-County, and Mwachinga and Dumbule in Kinango sub-County. During sample collection (stool, urine, and blood), informed consent forms were signed by all participating WRA or their guardians for those below the age of eighteen.

\section{Socio-demographic information}

Socio-demographic data was obtained from all pregnant and non-pregnant women using a pre-tested questionnaire (Supplementary file 1). Data collection was done by trained field assistants using android based mobile phones where the questionnaires had been programmed into using Open Data Kit (ODK) software. ODK [44] incorporates in-built data quality checks to reduce data entry errors making it very attractive to use.

\section{Parasitological examination}

S. haematobium infection was determined by examining fresh urine samples using nuclear pore filtration technique $[45,46]$. Briefly, $10 \mathrm{ml}$ of urine collected from WRA between $10 \mathrm{am}$ and $2 \mathrm{pm}$ was filtered in duplicate through $12.0 \mu \mathrm{m}(13 \mathrm{~mm})$ polycarbonate membrane filters (Sterlitech, Kent, WA, USA) mounted on urine filtration chambers. The membranes were then placed on labeled slides and examined using a microscope. Mean egg counts were calculated and expressed as egg counts per $10 \mathrm{ml}$ urine, then categorized as either light ( $\leq 50 \mathrm{eggs} / 10 \mathrm{ml}$ urine) or heavy intensity ( $>50$ eggs $/ 10 \mathrm{ml}$ urine) according to WHO guidelines [47].

The presence or absence of STH ova in stool was determined using Kato-Katz method. Briefly, duplicate thick smears were prepared from each stool sample using a sieve and a template calibrated to contain $41.7 \mathrm{mg}$ of stool and covered with cellophane strips pre-soaked in glycerol-malachite green solution. The slides were examined for STH ova using a microscope. Egg counts were multiplied by a factor of 24 to obtain the eggs per gram of stool and categorized as light, moderate, or heavy intensity as per the WHO guidelines [47].

For malaria examination, a drop of venous blood was used to make thick and thin blood smears which were 


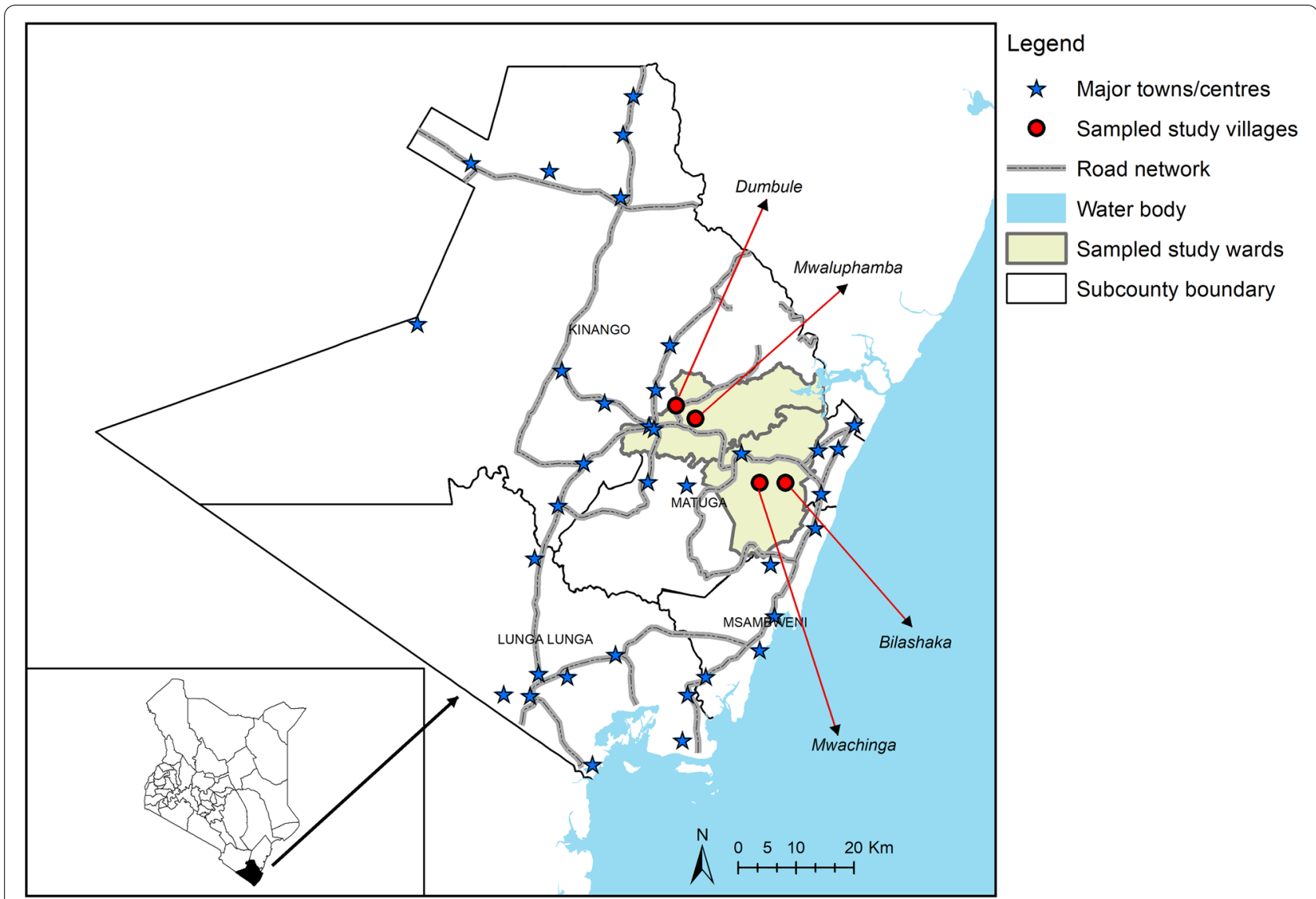

Fig. 1 Study site: A map of the study site showing the four villages; Bilashaka, Mwaluphamba, Dumbule, and Mwachinga

prepared and stained with $2 \%$ Giemsa for $30 \mathrm{~min}$, then washed in distilled water for one minute and examined under a microscope by an experienced laboratory technologist. Thick smears were used to identify parasite densities where malaria parasites were reported against 200 white blood cells counted, while thin blood smears were used to identify the species that is specific of the malaria causing parasites observed under thick blood smears. A senior technologist examined $10 \%$ of all the blood smear slides for quality assurance and quality control. Any discrepancies were repeated and reconciled then captured electronically on laboratory reporting data forms that had been programmed onto android based smart phones using ODK [44].

\section{Statistical analysis}

Prevalence of malaria, schistosomiasis and STH were calculated and the 95\% confidence intervals (CIs) determined using binomial regression model. Average intensities were determined for schistosomiasis and STH and the associated $95 \%$ CIs estimated using negative binomial regression model. The prevalence risk ratio (RR) and the incidence rate ratio (IRR) for binomial and negative binomial regression models respectively were reported. Risk factor analysis was conducted for malaria, schistosomiasis, and STH in a two-step approach. Univariable analysis was conducted using multi-level mixed effects logistic regression model at two levels; participants nested within villages selected from sub-counties in Kwale County. In this analysis, estimates were described as odds ratios (OR). Thereafter, multivariable analysis was performed using sequential block-wise approach for each outcome of interest, where the selected covariates were included and eliminated one at a time until the most parsimonious model was obtained. The adjusted odds ratios $(\mathrm{aOR})$ were obtained by mutually adjusting all minimum generated variables in a multi-level mixed effects logistic regression model at two levels. Prior to performing multivariable analysis, all covariates that met the inclusion criteria of $p<0.09$ were investigated for collinearity using pairwise correlation. A $p$-value of $\leq 0.05$ was considered statistically significant. All the statistical analyses were carried out using STATA version 15.1 (STATA Corporation, College Station, TX, USA). 


\section{Results}

Socio-demographic characteristics of the study population Overall, the data was collected from 534 WRA from the sub-Counties of Matuga (251 participants) and Kinango (283 participants). Two villages were sampled from each sub-county. The mean age of the participants was 30 years (range: $15-50$ years, standard deviation (SD): 9 years). Majority of the participants were aged between 21 and 30 years, 220 (41.2\%), followed by those aged 31 to 40 years, 149 (27.9\%). The demographic characteristics of the study population are summarized in Table 1.

\section{Pregnancy}

Of all the surveyed participants, 467 (87.5\%) reported that they had been pregnant before, and the reported average age at first pregnancy was 19.4 years (range: 13-35 and SD: 3.2 years). The average number of past pregnancies was 4.4 (range: $1-13$, SD: 2.5 pregnancies), with average live births of 3.9 (range: $1-10$ and SD: 2.1 live births). Further, all the participants were tested to confirm their current pregnancy status. From the results, 77 (14.5\%) were found to be pregnant (Table 1). Being pregnant was associated with increased odds of $S$. haematobium infection (OR=2.69, $p=0.007$ ) (Table 2$),(\mathrm{aOR}=2.77, p=0.015)$ (Table 3$)$. Pregnancy was also associated with increased odds of malaria infection $(\mathrm{OR}=3.40, p=0.016)$ (Table 2$)(\mathrm{aOR}=3.33$, $p<0.001$ ) (Table 4).

\section{STH and S. haematobium prevalence and mean intensity}

Overall, prevalence for any STH infection was 5.6\% (95\% CI: 2.8-11.3), with highest infection observed in Matuga sub-County $8.8 \%$ (95\% CI: 6.3-12.3), followed by Kinango sub-County $2.8 \%$ (95\% CI: 0.7-12.2). For specific STH species, the overall prevalence was $5.3 \%$ (95\% CI: 2.5-10.9) for hookworm and 0.6\% (95\% CI: $0.2-1.9)$ for $T$. trichiura. The overall mean intensity was 1414 eggs (95\% CI: 547-3654) and 52 eggs per gram of stool (95\% CI: 17-162) for hookworm and T. trichiura respectively. For the two species, mean intensities of infection were categorized as light intensity (Table 5).

The overall prevalence of S. haematobium was $3.8 \%$ (95\% CI: 2.6-5.4) with the highest infections observed in Kinango sub-County 4.3\% (95\% CI: 2.2-8.3), followed by Matuga sub-County 3.2\% (95\% CI: 2.7-3.8) (Table 5). The overall mean intensity of infection was 63 eggs/10 ml (95\% CI: 36-112), and 18/542 (3.4\%) had light infections while $2 / 542(0.4 \%)$ had heavy infections. The highest mean intensity was recorded in Matuga sub-County with 91 eggs/10 ml (95\% CI: 64-129) where $6 / 259$ (2.4\%) had light infections while $2 / 259$ (0.8\%) had heavy infections followed by Kinango sub-County with
39 eggs/10 ml (95\% CI: 13-118) where all infections were of light intensity.

Prevalence of $S$. haematobium was highest in women aged below 20years 6.0\% (95\% CI: 3.5-10.2), followed by those aged between 21 and 30years, 5.1\% (95\% CI: 2.9-8.9) and those between 31 and 40 years $2.0 \%$ (95\% CI: 0.5-7.8) (Table 5). There were no cases of any parasites among those aged between 41 and 50 years.

Amongst the pregnant women, prevalence of $S$. haematobium was $7.9 \%$ (95\% CI: 4.1-15.2) while that for nonpregnant women was $3.1 \%(2.0-4.7)$. A high prevalence of $S$. haematobium was observed among women who had one pregnancy in their lifetime $4 / 61(6.6 \%)$ as compared to those who had two pregnancies $3 / 65$ (4.6\%) and more than two pregnancies $10 / 339$ (2.9\%).

\section{Malaria prevalence and bed net usage}

The overall prevalence of malaria was $4.9 \%$ (95\% CI: 2.0-11.7) (Table 6), with the highest infections occurring in Matuga sub-County at 7.9\% (95\% CI: 3.5-18.0), followed by Kinango sub-County at 2.1\% (95\% CI: 2.1-2.1). The prevalence of malaria among pregnant women was 11.7\% (95\% CI: 3.6-37.5) and 3.7\% (95\% CI: 1.7-8.2) among non-pregnant women. Further, infection with malaria was highest among the younger women aged below 20years with a prevalence of $8.1 \%$ (95\% CI: $2.1-$ $31.5)$, followed by those aged 21 to 30 years at $5.0 \%(95 \%$ CI: 1.9-12.9) (Table 6). Bed net ownership and usage among pregnant women was high, 58/66 (87.8\%) and $56 / 58$ (96.6\%), respectively, compared to those who were not pregnant. Of all participants who reported owning at least a bed net, $7 / 58(12.1 \%)$ were infected with malaria, compared to the $2 / 8(25.0 \%)$ who reportedly did not own at least a bed net and were infected.

\section{Co-infections with S. haematobium, STH, and malaria infections}

The occurrence of co-infection was low and was recorded between S. haematobium and P. falciparum (0.6\% (95\% CI: $0.2-2.0)$ ), followed by that of $S$. haematobium and STH (0.4\% (95\% CI: 0.1-1.1)). However, there was no co-infection between malaria and STH. Additionally, none of the participants were co-infected with all the three infections.

Among the pregnant women, 2.6\% (95\% CI: 0.7-10.3) had co-infection of $S$. haematobium and $P$. falciparum and only $1.3 \%$ (95\% CI: $0.2-10.3)$ had co-infection of $S$. haematobium and hookworm or T. trichiura. Among the non-pregnant women, co-infection of S. haematobium and P. falciparum was $0.2 \%$ (95\% CI: $0-1.5 \%)$. Similarly, co-infection of S. haematobium and hookworm or $T$. trichiura was $0.2 \%$ (95\% CI: $0-1.5 \%)$. 
Table 1 Reported individual and household characteristics and the number of individuals positive for STH, S. haematobium and malaria infections

\begin{tabular}{|c|c|c|c|c|c|c|}
\hline \multirow[t]{2}{*}{ Factor } & \multirow{2}{*}{$\begin{array}{l}\text { Number examined } \\
(\%)\end{array}$} & \multicolumn{3}{|l|}{ STH infection ${ }^{a}$} & \multirow[t]{2}{*}{ S. haematobium } & \multirow[t]{2}{*}{ Malaria } \\
\hline & & STH combined & Hookworm & T. trichiura & & \\
\hline Overall & $534(100.0 \%)$ & $30(5.6 \%)$ & $28(5.3 \%)$ & $3(0.6 \%)$ & $20(3.8 \%)$ & $26(4.9 \%)$ \\
\hline \multicolumn{7}{|l|}{ Demographic characteristics } \\
\hline \multicolumn{7}{|l|}{ Age groups } \\
\hline 20 years and below & $100(18.7 \%)$ & $4(4.0 \%)$ & $4(4.0 \%)$ & 0 & $6(6.0 \%)$ & $8(8.1 \%)$ \\
\hline $21-30$ years & $220(41.2 \%)$ & $14(6.4 \%)$ & $14(6.4 \%)$ & $1(0.5 \%)$ & $11(5.1 \%)$ & $11(5.0 \%)$ \\
\hline $31-40$ years & $149(27.9 \%)$ & $11(7.4 \%)$ & $10(6.7 \%)$ & $1(0.7 \%)$ & $3(2.0 \%)$ & $4(2.7 \%)^{*}$ \\
\hline $41-50$ years & $65(12.2 \%)$ & $1(1.6 \%)$ & 0 & $1(1.6 \%)$ & 0 & $3(4.6 \%)$ \\
\hline \multicolumn{7}{|l|}{ Level of education } \\
\hline None & $151(28.3 \%)$ & $12(7.9 \%)$ & $12(7.9 \%)$ & $1(0.7 \%)$ & $6(3.9 \%)$ & $6(3.9 \%)$ \\
\hline Primary & $313(59.7 \%)$ & $18(5.8 \%)$ & $16(5.1 \%)$ & $2(0.6 \%)$ & $11(3.6 \%)$ & $18(5.8 \%)$ \\
\hline Secondary & $60(11.2 \%)$ & 0 & 0 & 0 & $3(5.0 \%)$ & $2(3.4 \%)$ \\
\hline Post-secondary & $10(1.9 \%)$ & 0 & 0 & 0 & 0 & 0 \\
\hline \multicolumn{7}{|l|}{ Average income (Ksh) } \\
\hline Below 15,000 & $461(86.3 \%)$ & $23(5.0 \%)$ & $21(4.6 \%)$ & $3(0.7 \%)$ & $17(3.7 \%)$ & $22(4.8 \%)$ \\
\hline Between 15,000-30,000 & $52(9.7 \%)$ & $5(9.6 \%)$ & $5(9.6 \%)$ & 0 & $3(5.8 \%)$ & $2(3.9 \%)$ \\
\hline Above 30,000 & $21(3.9 \%)$ & $2(9.5 \%)$ & $2(9.5 \%)$ & 0 & 0 & $2(9.5 \%)$ \\
\hline \multicolumn{7}{|l|}{ Marital status } \\
\hline Single/divorced/widowed & $121(22.7 \%)$ & $5(4.1 \%)$ & $5(4.1 \%)$ & 0 & $4(3.3 \%)$ & $5(4.2 \%)$ \\
\hline Married & $413(77.3 \%)$ & $25(6.1 \%)$ & $23(5.6 \%)$ & $3(0.7 \%)$ & $16(3.9 \%)$ & $21(5.1 \%)$ \\
\hline \multicolumn{7}{|l|}{ Occupation } \\
\hline Farmer & $195(36.5 \%)$ & $12(6.2 \%)$ & $12(6.2 \%)$ & 0 & $9(4.6 \%)$ & $9(4.6 \%)$ \\
\hline Business & $99(18.5 \%)$ & $7(7.1 \%)$ & $7(7.1 \%)$ & 0 & $4(4.0 \%)$ & $6(6.1 \%)$ \\
\hline Housewife/No job & $177(33.2 \%)$ & $7(3.9 \%)$ & $5(2.8 \%)^{*}$ & $3(1.7 \%)$ & $5(2.9 \%)$ & $10(5.7 \%)$ \\
\hline Salaried worker & $9(1.7 \%)$ & 0 & 0 & 0 & 0 & 0 \\
\hline Casual laborer & $23(4.3 \%)$ & $3(13.0 \%)^{*}$ & $3(13.0 \%)^{*}$ & 0 & $2(8.7 \%)$ & $1(4.4 \%)$ \\
\hline Others & $31(5.8 \%)$ & $1(3.2 \%)$ & $1(3.2 \%)$ & 0 & 0 & 0 \\
\hline \multicolumn{7}{|l|}{ Religion } \\
\hline Christian & $52(9.7 \%)$ & $4(7.7 \%)$ & $4(7.7 \%)$ & 0 & $3(5.8 \%)$ & $3(5.8 \%)$ \\
\hline Islam & $482(90.3 \%)$ & $26(5.4 \%)$ & $24(4.9 \%)$ & $3(0.6 \%)$ & $17(3.6 \%)$ & $23(4.8 \%)$ \\
\hline \multicolumn{7}{|l|}{ Pregnancy factors } \\
\hline \multicolumn{7}{|l|}{ Pregnancy result } \\
\hline Positive & $77(14.5 \%)$ & $4(5.2 \%)$ & $4(5.2 \%)$ & 0 & $6(7.9 \%)^{* *}$ & $9(11.7 \%)^{* * *}$ \\
\hline Negative & $455(85.5 \%)$ & $25(5.5 \%)$ & $23(5.1 \%)$ & $3(0.7 \%)$ & $14(3.1 \%)$ & $17(3.7 \%)$ \\
\hline \multicolumn{7}{|l|}{ Age when first pregnant } \\
\hline$<18$ years & $127(27.2 \%)$ & $10(7.9 \%)$ & $9(7.1 \%)$ & $1(0.8 \%)$ & $4(3.2 \%)$ & $6(4.7 \%)$ \\
\hline $18-25$ years & $321(68.7 \%)$ & $18(5.6 \%)$ & $17(5.3 \%)$ & $2(0.6 \%)$ & $12(3.8 \%)$ & $17(5.3 \%)$ \\
\hline$>25$ years & $19(4.1 \%)$ & 0 & 0 & 0 & $1(5.3 \%)$ & $1(5.3 \%)$ \\
\hline \multicolumn{7}{|c|}{ Number of pregnancies in lifetime } \\
\hline One & $61(13.1 \%)$ & $2(3.3 \%)$ & $2(3.3 \%)$ & 0 & $4(6.6 \%)$ & $7(11.5 \%)$ \\
\hline Two & $67(14.4 \%)$ & $2(2.9 \%)$ & $2(2.9 \%)$ & $1(1.5 \%)$ & $3(4.6 \%)$ & $4(5.9 \%)$ \\
\hline More than two & $339(72.6 \%)$ & $24(7.1 \%)$ & $22(6.5 \%)$ & $2(0.6 \%)$ & $10(2.9 \%)$ & $13(3.8 \%)^{* *}$ \\
\hline \multicolumn{7}{|l|}{ Ownership of a mosquito net } \\
\hline Yes & $58(87.8 \%)$ & $4(6.9 \%)$ & $4(6.9 \%)$ & 0 & $5(8.8 \%)$ & $7(12.1 \%)$ \\
\hline No & $8(12.1 \%)$ & 0 & 0 & 0 & $1(12.5 \%)$ & $2(25.0 \%)$ \\
\hline \multicolumn{7}{|l|}{ Given iron } \\
\hline Yes & $35(53.0 \%)$ & $2(5.7 \%)$ & $2(5.7 \%)$ & 0 & $3(8.8 \%)$ & $4(11.4 \%)$ \\
\hline No & $31(46.9 \%)$ & $2(6.5 \%)$ & $2(6.5 \%)$ & 0 & $3(9.7 \%)$ & $5(16.1 \%)$ \\
\hline
\end{tabular}


Table 1 (continued)

\begin{tabular}{|c|c|c|c|c|c|c|}
\hline \multirow[t]{2}{*}{ Factor } & \multirow{2}{*}{$\begin{array}{l}\text { Number examined } \\
(\%)\end{array}$} & \multicolumn{3}{|l|}{ STH infection ${ }^{a}$} & \multirow[t]{2}{*}{ S. haematobium } & \multirow[t]{2}{*}{ Malaria } \\
\hline & & STH combined & Hookworm & T. trichiura & & \\
\hline \multicolumn{7}{|l|}{ Taken deworming tablets } \\
\hline Yes & $24(36.4 \%)$ & $2(8.3 \%)$ & $2(8.3 \%)$ & 0 & $2(8.3 \%)$ & $3(12.5 \%)$ \\
\hline No & $42(63.6 \%)$ & $2(4.8 \%)$ & $2(4.8 \%)$ & 0 & $4(9.8 \%)$ & $6(14.3 \%)$ \\
\hline \multicolumn{7}{|l|}{ Taken antimalarial tablets } \\
\hline Yes & $33(50.0 \%)$ & $2(6.1 \%)$ & $2(6.1 \%)$ & 0 & $2(6.1 \%)$ & $5(15.2 \%)$ \\
\hline No & $33(50.0 \%)$ & $2(6.1 \%)$ & $2(6.1 \%)$ & 0 & $4(12.5 \%)$ & $4(12.1 \%)$ \\
\hline \multicolumn{7}{|l|}{ Individual WASH factors } \\
\hline \multicolumn{7}{|l|}{ Handwash after helping child defecate } \\
\hline Always & $372(69.7 \%)$ & $20(5.4 \%)$ & $19(5.1 \%)$ & $2(0.5 \%)$ & $8(2.2 \%)$ & $14(3.8 \%)$ \\
\hline Sometimes & $140(26.2 \%)$ & $8(5.7 \%)$ & $7(5.0 \%)$ & $1(0.7 \%)$ & $10(7.1 \%)^{* *}$ & $10(7.1 \%)$ \\
\hline Never & $22(4.1 \%)$ & $2(9.1 \%)$ & $2(9.1 \%)$ & 0 & $2(9.1 \%)$ & $2(9.1 \%)^{*}$ \\
\hline \multicolumn{7}{|l|}{ Handwash before preparing food } \\
\hline Always & $370(69.3 \%)$ & $20(5.4 \%)$ & $20(5.4 \%)$ & $1(0.3 \%)$ & $11(2.9 \%)$ & $17(4.6 \%)$ \\
\hline Sometimes & $114(21.4 \%)$ & $8(7.0 \%)$ & $6(5.3 \%)$ & $2(1.8 \%)$ & $7(6.2 \%)$ & $7(6.2 \%)$ \\
\hline Never & $50(9.4 \%)$ & $2(4.0 \%)$ & $2(4.0 \%)$ & 0 & $2(4.1 \%)$ & $2(4.0 \%)$ \\
\hline \multicolumn{7}{|l|}{ Handwash after toilet use } \\
\hline Always & $471(88.2 \%)$ & $26(5.5 \%)$ & $25(5.3 \%)$ & $2(0.4 \%)$ & $18(3.9 \%)$ & $24(5.1 \%)$ \\
\hline Sometimes & $48(8.9 \%)$ & $2(4.2 \%)$ & $1(2.1 \%)$ & $1(2.1 \%)$ & $2(4.2 \%)$ & $2(4.2 \%)$ \\
\hline Never & $15(2.8 \%)$ & $2(13.3 \%)$ & $2(13.3 \%)$ & 0 & 0 & 0 \\
\hline \multicolumn{7}{|l|}{ Anal cleansing material used } \\
\hline Toilet paper & $7(1.3 \%)$ & $1(14.3 \%)$ & $1(14.3 \%)$ & $1(14.3 \%)$ & $1(14.3 \%)$ & $1(14.3 \%)$ \\
\hline Water & $521(97.6 \%)$ & $29(5.6 \%)$ & $27(5.2 \%)$ & $2(0.4 \%)$ & $19(3.7 \%)$ & $25(4.8 \%)$ \\
\hline Leaves & $6(1.1 \%)$ & 0 & 0 & 0 & 0 & 0 \\
\hline \multicolumn{7}{|l|}{ Household WASH factors } \\
\hline Improved water source for drinking & $354(66.3 \%)$ & $21(5.9 \%)$ & $20(5.7 \%)$ & $2(0.6 \%)$ & $14(3.9 \%)$ & $22(6.2 \%)$ \\
\hline Improved water source for household use & $274(51.3 \%)$ & $17(6.3 \%)$ & $16(5.9 \%)$ & $2(0.7 \%)$ & $12(4.4 \%)$ & $19(6.9 \%)^{*}$ \\
\hline Treatment of drinking water & $92(17.2 \%)$ & $5(5.4 \%)$ & $5(5.4 \%)$ & 0 & $6(6.6 \%)^{* *}$ & $5(5.4 \%)$ \\
\hline \multicolumn{7}{|l|}{ Methods for water treatment } \\
\hline Boiling & $21(22.8 \%)$ & $3(14.3 \%)$ & $3(14.3 \%)$ & 0 & $2(9.5 \%)$ & $1(4.8 \%)$ \\
\hline Chlorine/Bleach & $66(71.7 \%)$ & $2(3.0 \%)^{* * *}$ & $2(3.0 \%)^{* * *}$ & 0 & $4(6.2 \%)$ & $3(4.6 \%)$ \\
\hline Others & $5(5.4 \%)$ & 0 & 0 & 0 & 0 & $1(20.0 \%)$ \\
\hline Improved latrine facility & $417(78.1 \%)$ & $28(6.7 \%)^{* *}$ & $26(6.3 \%)^{*}$ & $3(0.7 \%)$ & $14(3.4 \%)$ & $20(4.8 \%)$ \\
\hline Shared latrine with other households & $197(36.9 \%)$ & $6(3.1 \%)$ & $5(2.5 \%)$ & $1(0.5 \%)$ & $4(2.1 \%)^{*}$ & $12(6.1 \%)$ \\
\hline \multicolumn{7}{|l|}{ Household assets } \\
\hline Electricity & $245(45.9 \%)$ & $10(4.1 \%)^{*}$ & $9(3.7 \%)^{*}$ & $1(0.4 \%)$ & $6(2.5 \%)$ & $11(4.5 \%)$ \\
\hline Radio & $265(49.6 \%)$ & $14(5.3 \%)$ & $12(4.6 \%)$ & $2(0.8 \%)$ & $10(3.8 \%)$ & $13(4.8 \%)$ \\
\hline Television & $64(11.9 \%)$ & $4(6.3 \%)$ & $3(4.7 \%)$ & $1(1.6 \%)$ & $2(3.1 \%)$ & $2(3.2 \%)$ \\
\hline Mobile phone & $481(90.1 \%)$ & $28(5.8 \%)$ & $26(5.4 \%)$ & $3(0.6 \%)$ & $17(3.6 \%)$ & $22(4.6 \%)$ \\
\hline Bank account & $103(19.3 \%)$ & $4(3.9 \%)$ & $4(3.9 \%)$ & 0 & $5(4.9 \%)$ & $4(3.9 \%)$ \\
\hline Agricultural land & $503(94.2 \%)$ & $28(5.6 \%)$ & $26(5.2 \%)$ & $3(0.6 \%)$ & $20(4.0 \%)$ & $24(4.8 \%)$ \\
\hline Cows and goats & $306(57.3 \%)$ & $16(5.3 \%)$ & $16(5.2 \%)$ & $1(0.3 \%)$ & $11(3.6 \%)$ & $14(4.6 \%)$ \\
\hline Chicken and ducks & 486 (91.0\%) & $28(5.8 \%)$ & $26(5.4 \%)$ & $3(0.6 \%)$ & $19(3.9 \%)$ & $22(4.5 \%)^{*}$ \\
\hline
\end{tabular}

${ }^{a}$ Ascarislumbricoides was not included in the analysis because no cases were recorded for this particular STH species

${ }^{*}$ indicates significance at $p \leq 0.05,{ }^{* *}$ indicates significance at $p \leq 0.01,{ }^{* * *}$ indicates significance at $p \leq 0.001$

Individual and household WASH factors

Over half of the women reported use of improved water sources for drinking $354(66.3 \%)$ with use of a public tap/stand pipe being the most common source (45.7\%). In Matuga and Kinango sub-counties, use of improved water sources for drinking was reportedly high at 
Table 2 Univariable analysis of factors associated with STH, S. haematobium or malaria

\begin{tabular}{|c|c|c|c|c|c|}
\hline \multirow[t]{2}{*}{ Factor } & \multirow{2}{*}{$\begin{array}{l}\text { Number examined } \\
(\%)\end{array}$} & \multicolumn{4}{|c|}{ Univariable logistic regression [OR $(95 \% \mathrm{Cl}) ; p$-value] } \\
\hline & & $\begin{array}{l}\text { STH combined } \\
(n=30)\end{array}$ & $\begin{array}{l}\text { Hookworm } \\
(n=28)\end{array}$ & $\begin{array}{l}\text { S. haematobium } \\
(n=20)\end{array}$ & $\begin{array}{l}\text { Malaria } \\
(n=26)\end{array}$ \\
\hline \multicolumn{6}{|l|}{ Demographic characteristics } \\
\hline \multicolumn{6}{|l|}{ Age groups } \\
\hline 20 years and below & $100(18.7 \%)$ & Reference & & & \\
\hline $21-30$ years & $220(41.2 \%)$ & $1.63, p=0.362$ & $1.63, p=0.362$ & $0.84, p=0.345$ & $0.60, p=0.496$ \\
\hline $31-40$ years & $149(27.9 \%)$ & $1.91, p=0.210$ & $1.73, p=0.204$ & $0.32, p=0.172$ & $0.31, p=0.020^{*}$ \\
\hline $41-50$ years & $65(12.2 \%)$ & $0.38, p=0.314$ & - & - & $0.55, p=0.316$ \\
\hline \multicolumn{6}{|l|}{ Pregnancy factors } \\
\hline Currently pregnant & $77(14.5 \%)$ & $0.94, p=0.899$ & $1.03, p=0.954$ & $2.69, p=0.007^{*}$ & $3.40, p=0.000^{*}$ \\
\hline \multicolumn{6}{|l|}{ Age when first pregnant } \\
\hline$<18$ years & $127(27.2 \%)$ & Reference & & & \\
\hline $18-25$ years & $321(68.7 \%)$ & $0.69, p=0.450$ & $0.73, p=0.623$ & $1.20, p=0.824$ & $1.13, p=0.686$ \\
\hline$>25$ years & $19(4.1 \%)$ & - & - & $1.71, p=0.090$ & $1.12, p=0.868$ \\
\hline \multicolumn{6}{|l|}{ Number of pregnancies } \\
\hline One & $61(13.1 \%)$ & Reference & & & \\
\hline Two & $67(14.4 \%)$ & $0.91, p=0.737$ & $0.91, p=0.737$ & $0.69, p=0.771$ & $0.49, p=0.307$ \\
\hline More than two & $339(72.6 \%)$ & $2.25, p=0.106$ & $2.05, p=0.171$ & $0.43, p=0.279$ & $0.31, p=0.008^{*}$ \\
\hline \multicolumn{6}{|l|}{ Bednet use and ownership } \\
\hline Net ownership & $58(87.8 \%)$ & - & - & $0.67, p=0.797$ & $0.41, p=0.318$ \\
\hline \multicolumn{6}{|l|}{ Net usage } \\
\hline Always & $56(96.6 \%)$ & - & - & $0.08, p=0.202$ & - \\
\hline Sometimes & $2(3.5 \%)$ & Reference & & & \\
\hline \multicolumn{6}{|l|}{ Individual WASH factors } \\
\hline \multicolumn{6}{|l|}{ Handwash after helping child defecate } \\
\hline Always & $372(69.7 \%)$ & Reference & & & \\
\hline Sometimes & $140(26.2 \%)$ & $1.06, p=0.928$ & $0.98, p=0.968$ & $3.47, p=0.006^{*}$ & $1.96, p=0.160$ \\
\hline Never & $22(4.1 \%)$ & $1.76, p=0.657$ & $1.85, p=0.641$ & $4.51, p=0.106$ & $2.55, p=0.021 *$ \\
\hline \multicolumn{6}{|l|}{ Handwash before preparing food } \\
\hline Always & $370(69.3 \%)$ & Reference & & & \\
\hline Sometimes & $114(21.4 \%)$ & $1.32, p=0.605$ & $0.97, p=0.947$ & $2.15, p=0.071$ & $1.37, p=0.559$ \\
\hline Never & $50(9.4 \%)$ & $0.73, p=0.757$ & $0.73, p=0.757$ & $1.38, p=0.542$ & $0.87, p=0.814$ \\
\hline \multicolumn{6}{|l|}{ Handwash after toilet use } \\
\hline Always & $471(88.2 \%)$ & Reference & & & \\
\hline Sometimes & $48(8.9 \%)$ & $0.74, p=0.769$ & $0.38, p=0.326$ & $1.09, p=0.910$ & $0.81, p=0.629$ \\
\hline Never & $15(2.8 \%)$ & $2.63, p=0.267$ & $2.74, p=0.232$ & - & - \\
\hline \multicolumn{6}{|l|}{ Household WASH factors } \\
\hline Improved water source for drinking & $354(66.3 \%)$ & $1.20, p=0.714$ & $1.29, p=0.627$ & $1.20, p=0.674$ & $2.92, p=0.231$ \\
\hline Improved water source for household use & $274(51.3 \%)$ & $1.26, p=0.596$ & $1.29, p=0.596$ & $1.45, p=0.460$ & $2.70, p=0.012^{*}$ \\
\hline Treatment of drinking water & $92(17.2 \%)$ & $0.96, p=0.842$ & $1.04, p=0.813$ & $2.15, p=0.003^{*}$ & $1.15, p=0.875$ \\
\hline \multicolumn{6}{|l|}{ Methods for water treatment } \\
\hline Boiling & $21(22.8 \%)$ & Reference & & & \\
\hline Chlorine/Bleach & $66(71.7 \%)$ & $0.19, p<0.001 *$ & $0.19, p<0.001^{*}$ & $0.62, p=0.577$ & $0.95, p=0.905$ \\
\hline Others & $5(5.4 \%)$ & - & - & - & $5.00, p=0.286$ \\
\hline Improved latrine facility & $417(78.1 \%)$ & $4.15, p=0.009^{*}$ & $3.83, p=0.021^{*}$ & $0.64, p=0.078$ & $0.93, p=0.915$ \\
\hline Shared latrine with other households & $197(36.9 \%)$ & $0.41, p=0.068$ & $0.35, p=0.071$ & $0.42, p=0.016^{*}$ & $1.49, p=0.242$ \\
\hline
\end{tabular}

*Indicates a statistically significant association

- variable omitted because of insufficient number of observations 
Table 3 Multivariable analysis of factors associated with $S$. haematobium

\begin{tabular}{|c|c|}
\hline Factor & $\begin{array}{l}\text { Multivariable } \\
\text { analysis [aOR, } \\
p \text {-value] } \\
(n=20)\end{array}$ \\
\hline Currently pregnant & $2.77, p=0.015^{*}$ \\
\hline \multicolumn{2}{|c|}{ Washing hands after helping a child defecate } \\
\hline Always & Reference \\
\hline Sometimes & $4.03, p=0.003^{*}$ \\
\hline Never & $5.62, p=0.078$ \\
\hline Treatment of drinking water & $3.55, p=0.003^{*}$ \\
\hline Improved latrine & $0.69, p=0.037^{*}$ \\
\hline
\end{tabular}

*Indicates a statistically significant association

Table 4 Multivariable analysis of factors associated with malaria infection

\begin{tabular}{|c|c|}
\hline Factor & $\begin{array}{l}\text { Multivariable } \\
\text { analysis [aOR, } \\
p \text {-value] } \\
(n=26)\end{array}$ \\
\hline Currently pregnant & $3.33, p<0.001^{*}$ \\
\hline \multicolumn{2}{|l|}{ Number of pregnancies } \\
\hline One & Reference \\
\hline Two & $0.39, p=0.213$ \\
\hline More than two & $0.44, p=0.055$ \\
\hline Improved water sources for household sources & $3.24, p<0.001^{*}$ \\
\hline Owning chicken and ducks & $0.37, p=0.028^{*}$ \\
\hline \multicolumn{2}{|l|}{ Washing hands after helping a child defecate } \\
\hline Always & Reference \\
\hline Sometimes & $1.59, p=0.298$ \\
\hline Never & $3.23, p<0.001 *$ \\
\hline
\end{tabular}

*Indicates a statistically significant association

$178 / 251(70.9 \%)$ and 176/283 (62.2\%) respectively. Only $92(17.2 \%)$ of the participants reportedly treated their water to make it safer for drinking. The commonly used methods for treating drinking water were; chlorination/bleach 66/92 (71.7\%) and boiling 21/92 (22.8\%). Treating of drinking water using chlorine was shown to reduce the odds of $\mathrm{STH}$ infection $(\mathrm{OR}=0.19, p=0.001)$ (Table 2$),(\mathrm{aOR}=0.27, p=0.001)$ (data not shown).

Use of an improved sanitation facility was reported by majority of the participants 417 (78.1\%) and the most common sanitation type was the traditional pit latrine 328 (61.4\%). Matuga sub-County, reported the highest use of improved sanitation facilities (231/251 (92.0\%)) followed by Kinango sub-County (186/283 (65.7\%)). Less than half of the participants reported sharing a latrine facility with other households 197 (36.9\%). Use of a shared latrine facility was highest in Matuga
sub-County (95/251 (37.9\%)) compared to Kinango sub-County (102/283 (36.0\%)).

Handwashing was reportedly mostly done after helping a child to defecate with $372(69.6 \%)$ of the women reporting to always wash their hands while $140(26.2 \%)$ reported washing their hands sometimes and 22 (4.1\%) reported never washing their hands. Before preparing food, $370(69.3 \%)$ reported that they always washed their hands, $114(21.4 \%)$ reported washing hands sometimes while $50(9.4 \%)$ did not wash their hands. After visiting the toilet, $471(88.2 \%)$ reported to always wash their hands, $48(8.9 \%)$ washed their hands sometimes and 15 $(2.8 \%)$ never washed their hands.

\section{Discussion}

Overall, the prevalence for any STH infection was 5.6\% with the highest infection observed in Matuga subCounty at $8.8 \%$ compared to Kinango sub-County that had a prevalence of $2.8 \%$. It is not clear why there was a variance in prevalence between these two sub-counties since demographic characteristics and WASH factors were similar. In fact, Matuga sub-County reported the highest use of improved sanitation facilities (92.0\%) compared to Kinango sub-County (65.7\%). Several studies [48-50] have shown a strong association between geophagy and STH infections and the difference in prevalence observed in Matuga and Kinango sub-Counties might be attributed to possible behavior differences in this regard. However, our study did not investigate geophagy factors. The overall prevalence for hookworm and T. trichiura were 5.3 and $0.6 \%$ respectively. Both of the STH species reported had light intensities. Compared to previous studies $[16,26,51,52]$, this prevalence was very low. A study done in Mwaluphamba, in Kwale County in 2010 found a high prevalence of hookworm (41.7\%) among adults [11]. The drastic reduction in prevalence we found may be attributed to the National School Based Deworming Programme which started in 2012 up to 2017 [53, 54] targeting school age children. It may also be attributed to the TUMIKIA project which was conducted in Kwale between 2015 and 2017 [23] where albendazole was distributed annually and bi-annually both in school based and community based MDA. However, preventive chemotherapy strategies have been shown to end up with re-infections thereby reducing the gains by such programs [54]. In addition, it has been reported in other studies that the prevalence of STH infections may remain high even after mass drug administration through school based deworming programs [16, 51, 52]. Since the Mwaluphamba study that recorded a prevalence of $41.7 \%$ of hookworm among adults was done several years ago, it is possible that sanitation, health education, behavior change including use of toilets and water 
Table 5 Prevalence and mean intensity of STH and S. haematobium infections by sub-county and village

\begin{tabular}{|c|c|c|c|c|}
\hline \multirow[t]{2}{*}{ Villages } & \multicolumn{3}{|l|}{ STH infection ${ }^{a}$} & \multirow{2}{*}{$\begin{array}{l}\text { S. haematobium } \\
(n=20)\end{array}$} \\
\hline & $\begin{array}{l}\text { STH combined } \\
(n=30)\end{array}$ & $\begin{array}{l}\text { Hookworms } \\
(n=28)\end{array}$ & $\begin{array}{l}\text { T. trichiura } \\
(n=3)\end{array}$ & \\
\hline \multicolumn{5}{|c|}{ Prevalence of infection, $\%(95 \% \mathrm{Cl})$} \\
\hline Kinango sub-County & $2.8(0.7-12.2)$ & $2.5(0.6-9.9)$ & $0.7(0.1-4.9)$ & $4.3(2.2-8.3)$ \\
\hline Dumbule village & $0.7(0.1-4.9)$ & $0.7(9.1-4.9)$ & 0 & $2.8(1.1-7.5)$ \\
\hline Mwachinga village & $4.9(2.4-10.2)$ & $4.2(1.9-9.2)$ & $1.4(0.4-5.6)$ & $5.7(2.9-11.2)$ \\
\hline Matuga sub-County & $8.8(6.3-12.3)$ & $8.4(5.4-13.0)$ & $0.4(0.1-2.4)$ & $3.2(2.7-3.8)$ \\
\hline Bilashaka village & $10.4(6.1-17.8)$ & $10.4(6.1-17.8)$ & 0 & $3.5(1.3-9.2)$ \\
\hline Mwaluphamba village & $7.4(4.1-13.4)$ & $6.7(3.5-12.5)$ & $0.7(0.1-5.2)$ & $2.9(1.1-7.7)$ \\
\hline Overall prevalence & $5.6(2.8-11.3)$ & $5.3(2.5-10.9)$ & $0.6(0.2-1.9)$ & $3.8(2.6-5.4)$ \\
\hline \multicolumn{5}{|c|}{ Mean intensity of infection, epg $(95 \% \mathrm{Cl})$} \\
\hline Kinango sub-County & - & $496(95-2587)$ & $59(8-419)$ & $39(13-118)$ \\
\hline Dumbule & - & $77(1-11,467)$ & 0 & $17(3-93)$ \\
\hline Mwachinga & - & $913(108-7699)$ & 0 & $61(16-228)$ \\
\hline Matuga sub-County & - & $2453(1114-5403)$ & $43(7-262)$ & $91(64-129)$ \\
\hline Bilashaka & & 0 & 0 & $108(12-962)$ \\
\hline Mwaluphamba & & $1538(273-8666)$ & 0 & $76(9-639)$ \\
\hline Overall mean intensity & - & $1414(547-3654)$ & $52(17-162)$ & $63(36-112)$ \\
\hline
\end{tabular}

${ }^{a}$ Ascaris lumbricoides was not included in the analysis because no cases were recorded for this particular STH species

sources has improved ever since and therefore this might account for the low prevalence we observed in the current study. Indeed, our study found the use of improved water sources for drinking with use of public tap/stand pipe being the most common source. Use of improved

Table 6 Prevalence of malaria infections by sub-county and village

\begin{tabular}{ll}
\hline Categories & $\begin{array}{l}\text { Prevalence \%(95\%Cl) } \\
(\boldsymbol{n}=\mathbf{2 6})\end{array}$ \\
\hline Overall & $\mathbf{4 . 9}(\mathbf{2 . 0 - 1 1 . 7 )}$ \\
Kinango sub-County & $\mathbf{2 . 1}(\mathbf{2 . 1}-\mathbf{2 . 1})$ \\
Dumbule village & $2.1(0.7-6.5)$ \\
Mwachinga village & $2.1(0.7-6.5)$ \\
Matuga sub-County & $\mathbf{7 . 9}(\mathbf{3 . 5}-\mathbf{1 8 . 0})$ \\
Bilashaka village & $4.3(1.8-10.2)$ \\
Mwaluphamba village & $11.0(6.8-17.8)$ \\
By age group & \\
20years and below & $8.1(2.1-31.5)$ \\
21-30years & $5.0(1.9-12.9)$ \\
31-40years & $2.7(0.8-8.9)$ \\
$\quad 41-50 y e a r s$ & $4.6(1.6-12.9)$ \\
Bednet ownership & \\
$\quad$ Yes & $12.1(2.8-51.1)$ \\
No & $25.0(16.4-38.2)$ \\
Pregnancy result & \\
Positive & $11.7(3.6-37.5)$ \\
Negative & $3.7(1.7-8.2)$ \\
\hline
\end{tabular}

sanitation facilities was also reported with the most common sanitation type being the traditional pit latrine. This view is supported by another study which reported that the availability and use of sanitation facilities was associated with a reduction in the prevalence of soil transmitted helminthiasis [15]. However, this same study also noted that preventive chemotherapy appeared to be the main strategy to control STH infections in highly endemic areas.

Our study found a general trend of increase in STH infection with increase in age except for those aged between 41 to 50 years who recorded the lowest prevalence at $1.6 \%$. There was no significant difference of STH prevalence among pregnant women (5.2\%) compared to non-pregnant women (5.5\%). However, women who had more than two pregnancies in their lifetime had a high STH prevalence (7.1\%) than those who had one pregnancy. Increased odds of infection were found among those who used improved latrine facilities and casual laborers. This shows that STH infection may have resulted from water contamination rather than use or lack thereof latrines. This is demonstrated by the fact that there were reduced odds of STH infection among those who were treating drinking water with chlorine $(\mathrm{OR}=0.19 ; p=0.001)$. Taken together, the results here point to parity and WASH factors as significant determinants of STH infection status in Kinango and Matuga sub-counties. This is supported by Aranzales et al and Campbell et al who 
showed that parity and WASH factors, respectively, are associated with STH infections $[55,56]$.

The prevalence for Schistosoma infection among the WRA in the study area was $3.8 \%$ with the highest infections observed in Kinango sub-County at $4.3 \%$, followed by Matuga sub-County at $3.2 \%$. The overall observed prevalence here is lower compared to two studies done several years ago in Mwaluphamba in Kwale County; Kihara et al that showed a prevalence of $36.94 \%$ among pregnant and non-pregnant women [26] while Njenga et al which showed a prevalence of $18.2 \%$ among adults [11]. A number of other studies elsewhere [45, 52, 57$59]$ have also reported high prevalence of $S$. haematobium infections. S. haematobium infection rates have also been shown to be influenced by weather patterns, and local and focal clustering patterns [60] and this may partially explain the relatively low rates observed. Further, the socio-demographic factors observed might have led to a change of the study area from high risk to a low risk site. This speculation is supported by a study done in Tanzania which found a low prevalence of $4 \%$ among women of reproductive age in low risk sites compared to 53\% in high risk sites [61].

In the present study, younger women were more infected than older women and as age increased, $S$. haematobium infection rate reduced. Acquired protective immunity among older women might account for this difference. It could also be possible that younger women are doing most of the chores related to water contact such as washing clothes and fetching water and therefore are more exposed to the infection. Pregnancy was associated with increased odds of $S$. haematobium infection. A high prevalence of S. haematobium was observed among women who had one pregnancy in their lifetime (6.6\%) compared to those who had two pregnancies (2.9\%). This is different from another study that found no significant difference in the prevalence of urogenital schistosomiasis among pregnant and nonpregnant women in Kwale [26]. Our findings are, however, similar to a study done in Nigeria which showed that urogenital schistosomiasis among pregnant women was high at a prevalence of $20.8 \%$ with younger women and those pregnant at the greatest risk [62]. Similarly, the average prevalence of schistosomiasis in pregnant women in Anambra state, Nigeria, was reported to be high at $23.8 \%$ with younger (16 to 20 years old) pregnant women reported to have a higher prevalence of schistosomiasis than older pregnant women [63]. Improved usage of latrine facilities was associated with reduced odds of getting $S$. haematobium infection. Taken together, our results show that age, pregnancy, WASH factors, and poverty are risk factors contributing to $S$. haematobium infections among WRA in the current study which is in line with previous studies [55, $56,62,63]$.

The overall prevalence of malaria was $4.9 \%$ with the highest infections occurring in Matuga sub-County at $7.9 \%$ followed by Kinango sub-County at $2.1 \%$. Our results are similar to those by Njenga et. al. that found a malaria prevalence of $5.6 \%$ in Kwale [11]. The prevalence of malaria among pregnant and non-pregnant women was 11.7 and $3.7 \%$ respectively. The odds of getting malaria were statistically significantly higher for women who were at the time pregnant than those who were not pregnant. We speculate that this could be as a result of the differences in immune status between the two groups of women. This is supported by several studies as reviewed by Rogerson et al. [64]. More recent data supports this and has shown that antibody responses to pregnancy specific pRBCs and VAR2CSA antigens are not correlated with protection against malaria but rather markers of infection [65]. Additional findings in our study are that those who used improved water sources for drinking had increased odds of getting malaria. This is in contrast to another study that showed that improved water and sanitation conditions were associated with a decreased risk of malaria infection [66]. It is not clear why there was this association in our study. Further studies may be needed to explain this. Interestingly, owning chicken and ducks was statistically significantly associated with reduced odds of malaria infection. It has been shown that chicken volatile compounds act as medium to long range repellents of malaria causing Anopheles arabiensis thereby disrupting their host seeking behavior [67]. This could partially explain the observation that owning chicken and ducks reduced the odds of malaria infection. However, more work is needed to ascertain this. Those who had more than two pregnancies in their lifetime were less likely to have malaria $(\mathrm{OR}=0.31 ; p$ value $=0.008)$ unlike those with a single pregnancy. The prevalence of malaria was highest among younger women aged below 20 years $(8.1 \%)$ followed by those aged between 21 and 30 years at $5.0 \%$. This may be due to acquisition of protective immunity among the older population.

The occurrence of low co-infection rate between $S$. haematobium and P. falciparum (0.6\%) and that between $S$. haematobium and STH $(0.4 \%)$, could be attributed to the continuous school based deworming that has been taking place in the area for the past 7 years and the national insecticide treated bed net distribution and use by pregnant women for more than a decade. The low prevalence of co-infections found in this study compared to single infections is a trend that is supported by several studies. For example, a study done in Kingwede, Kwale County found that co-infection prevalence was lower than single infections and that children had 9.3 times the odds 
of co-infection compared to adults [68]. In another study by Malhotra et al [52], the prevalence of single infections was high compared to co-infection among mothers during antenatal clinic visits at Msambweni District Hospital. Similarly, a low prevalence of polyparasitism was recorded among pregnant women in Bogota, Colombia compared to single intestinal parasitism infections [56]. However, the trend was found to be different among children in a study done in Zambia which found a high prevalence of polyparasitism compared to single infections of malaria, hookworm, and S. haematobium [34].

There were no malaria co-infections with STH or with all the three parasitic infections in our study. This is contrary to a study by Florey et al which showed that the odds of S. haematobium infection increased as Plasmodium sp. infection intensity increased [68]. In another study done in Kwale, Njenga et al reported much higher prevalence of co-infections of S. haematobium with hookworm at $45.8 \%$ and that of S. haematobium with T. trichiura at 6.7\% [11]. Our study found that among those who were pregnant, $2.6 \%$ had co-infections with S. haematobium and malaria, while $1.3 \%$ had co-infections with $S$. haematobium and STH. Among non-pregnant women, co-infection with $S$. haematobium and malaria was $0.2 \%$ which was the same with co-infections of S. haematobium and STH. These results suggest that pregnant women are more susceptible to co-infections than non-pregnant women. It has long been believed that during pregnancy, the mothers' immunity is down regulated [64], which is supported by new data [65], and this may account for the disparity in prevalence among the pregnant and non-pregnant women.

This study had some limitation; firstly, the sensitivity of Kato Katz method of STH diagnosis may not pick low prevalence and intensity in an endemic area. A much more sensitive method [69] is thus recommended for future studies on prevalence and intensity of schistosomiasis and STH in the area under study. Secondly, we used a single day urine examination which may have led to missing some positive cases of S. haematobium [70] given the low prevalence and intensity observed in the study area. Further, given the low prevalence of parasitic infections in Matuga and Kinango sub-Counties, we needed a much larger sample size to make a more reasonable comparison of the different results; however, we were limited by the resources available for the study.

\section{Conclusion}

Our results generally show a low prevalence of STHs, S. haematobium, and malaria in Matuga and Kinango sub-counties with the common infection among the WRA that participated in this study being STH followed by malaria and then schistosomiasis. Light intensities were recorded for both STHs and S. haematobium.
Cases of co-infections were low, and occurred between schistosomiasis and malaria or STH. There were no coinfections recorded between malaria and STH. Regular school and community based treatment programs might have played a major role in reducing the STH and $S$. haematobium prevalence. Pregnant women were found to be disproportionately burdened by all the infections compared to non-pregnant women thereby supporting the fact that pregnancy is a significant risk factor for these infections in the study area. Overall, high cases of all the three infections were observed in Matuga subCounty compared to Kinango sub-County. In conclusion, our results show a general use of improved WASH factors in the study area that might have led to the low infection rates observed. Further, bed net ownership and usage were high and could have contributed to the low prevalence of malaria infections.

\begin{abstract}
Abbreviations
S. haematobium: Schistosoma haematobium; NTDs: Neglected Tropical Diseases; STH: Soil Transmitted Helminthes; T. trichiura: Trichuris trichiura; WRA :Women of Reproductive Age; S. mansoni: Schistosoma mansoni; S. japonicum: Schistosoma japonicum; OR: Odds Ratio; aOR: Adjusted Odds Ratio; WHO: World Health Organization; HIV-1: Human Immunodeficiency Virus strain 1; WASH: Water, Sanitation, and Hygiene; SBD: School Based Deworming; CBD: Community Based Deworming; MDA: Mass Drug Administration; TUMIKIA: Tuangamize Minyoo Kenya Imarisha Afya "together we eradicate worms in Kenya for a better health".
\end{abstract}

\section{Supplementary Information}

The online version contains supplementary material available at https://doi. org/10.1186/s12889-022-12526-0.

Additional file 1.

Additional file 2.

Acknowledgements

We are grateful to all the study participants and the Kwale County Government for approving the study. We thank all the research assistants involved in this study and the community health volunteers for the great job they did in mobilizing the community. We are indebted to the resident clinical officer at Mwaluphamba health facility, the nurses and community health workers at Bilashaka, Mwaluphamba, Mwachinga, and Dumbule health facilities for their support.

\section{Authors' contributions}

VTJ, FM, and JHK designed the study. VTJ, FM, LK, CM, JM, CO, HK, JK, ZN, and $J H K$ were involved in acquisition of the data. VTJ, CO, and JHK analyzed and interpreted the results. VTJ wrote the initial draft. VTJ, FM, LK, CM, JM, CO, HK, $J \mathrm{~K}, \mathrm{ZN}$, and JHK substantively revised the manuscript and approved the final version for submission.

\section{Funding}

This work was supported by the Kenya National Research Fund (grant no:; $\mathrm{NRF} / 1 / \mathrm{MMC} / 040$ ). The funding body had no role either in the design of the study or in the collection, analysis, interpretation of data, and in writing of the manuscript.

\section{Availability of data and materials}

The datasets used and/or analyzed during the current study are available from the corresponding author on reasonable request. 


\section{Declarations}

\section{Ethics approval and consent to participate}

The protocol for this study was reviewed and approved by the Scientific and Ethics Review Unit of Kenya Medical Research Institute (Ref No. KEMRI/SERU/ ESACIPAC/3684). The Kenya National Commission for Science, Technology, and Innovation (NACOSTI) certified the study according to the rules and regulations that govern field studies in Kenya. Permission to carry out the study in Kwale County was granted by the Kwale County Government through the County Ministry of Health offices. Participation in the study was by informed consent in either English or Swahili languages. Informed consent was obtained from parents or legal guardians for participants below the age of 18. For those who were illiterate, the consent was read to them by a legal guardian in Swahili language and their thumb print was used in place of a signature. All participants were aware they could withdraw from the study at any given time if they changed their minds. Those who turned positive for any of the three infections were referred for treatment at their nearest health facility free of charge.

\section{Consent for publication}

This study is published with permission from the Director of the Kenya Medical Research Institute.

\section{Competing interests}

The authors declare that they have no competing interests.

\section{Author details}

'Department of Medical Sciences, Technical University of Mombasa, Mombasa, Kenya. ${ }^{2}$ Department of Environment and Health Sciences, Technical University of Mombasa, Mombasa, Kenya. ${ }^{3}$ Center for Publich Health Research, Kenya Medical Research Institute, Nairobi, Kenya. ${ }^{4}$ Eastern and Southern Africa Center for International Parasite Control, Kenya Medical Research Institute, Nairobi, Kenya. ${ }^{5}$ Office of the Deputy Vice Chancellor, South Eastern Kenya University, Kitui, Kenya.

Received: 5 April 2021 Accepted: 6 January 2022

Published online: 19 January 2022

\section{References}

1. Colley DG. Human schistosomiasis. Lancet. 2014;383(9936):2253-64.

2. Vos T, Flaxman AD, Naghavi M, Lozano R, Michaud C, Ezzati M, et al. Years lived with disability (YLDs) for 1160 sequelae of 289 diseases and injuries 1990-2010: a systematic analysis for the global burden of disease study 2010. Lancet. 2012;380(9859):2163-96.

3. Bruun B, Aagaard-Hansen J, Watts S. The social context of schistosomiasis and its control an introduction and annotated bibliography. Geneva: WHO; 2008. p. VII.

4. Nour N. Schistosomiasis: health effects on women. Rev Obstet Gynaecol. 2010;3(1):28-32.

5. Friedman JF, Mital P, Kanzaria HK, Olds GR, Kurtis JD. Schistosomiasis and pregnancy. Trends Parasitol. 2007;23(4):159-64.

6. Tsoka-Gwegweni J, Ntombela N. A double load to carry: parasites and pregnancy. South Afr J Infect Dis. 2014;29(2):52-5.

7. Colley DG, Bustinduy AL, Secor WE, King CH. Human schistosomiasis. Lancet. 2014;368(9541):2253-64.

8. Friedman JF, Olveda RM, Mirochnick MH, Bustinduy AL, Elliott AM. Praziquantel for the treatment of schistosomiasis during human pregnancy. Bull World Health Organ. 2018;96(1):59-65.

9. Chitsulo L, Engels D, Montresor A, Savioli L. The global status of schistosomiasis and its control. Acta Trop [Internet]. 2000;77(1):41-51 Available from: https://www.ncbi.nlm.nih.gov/pmc/articles/PMC5633072/pdf/ emss-74305.pdf.

10. Clements ACA, Deville MA, Ndayishimiye O, Brooker S, Fenwick A. Spatial co-distribution of neglected tropical diseases in the east African Great Lakes region: revisiting the justification for integrated control. Tropical Med Int Health. 2010;15(2):198-207.

11. Njenga SM, Mwandawiro CS, Muniu E, Mwanje MT, Haji FM, Bockarie MJ. Adult population as potential reservoir of NTD infections in rural villages of Kwale district, coastal Kenya: implications for preventive chemotherapy interventions policy. Parasit Vectors. 2011;4(1):2-7.

12. Kaiglová A, Changoma MJS, Špajdelová J, Jakubcová D, Bírová K. Urinary schistosomosis in patients of rural medical health centers in Kwale county, Kenya. Helminthol. 2020;57(1):19-27.

13. Werkman M, Wright JE, Truscott JE, Oswald WE, Halliday KE, Papaiakovou $\mathrm{M}$, et al. The impact of community-wide, mass drug administration on aggregation of soil-transmitted helminth infection in human host populations. Parasit Vectors. 2020;13(1):1-12. https://doi.org/10.1186/ s13071-020-04149-4.

14. Lo NC, Addiss DG, Hotez PPJ, H PC, PJR S, Evans DS, et al. A call to strengthen the global strategy for schistosomiasis and soil-transmitted helminthiasis : the time is now. Lancet Infect Dis. 2017;17(2):e64-9.

15. Ziegelbauer K, Speich B, Mausezahl D, Bos R, Keiser J, Utzinger J. Effect of sanitation on soil-transmitted helminth infection: systematic review and Meta-analysis. PLoS Med. 2012;9(1):e1001162. https://doi.org/10.1371/ journal.pmed. 1001162.

16. Dunn JC, Bettis AA, Wyine NY, Lwin AMM, Tun A, Maung NS, et al. Soiltransmitted helminth reinfection four and six months after mass drug administration: results from the delta region of Myanmar. PLoS Negl Trop Dis. 2019;13(2):1-16. https://doi.org/10.1371/journal.pntd.0006591.

17. Wepnje GB, Anchang-Kimbi JK, Ndassi VD, Lehman LG, Kimbi HK. Schistosoma haematobium infection status and its associated risk factors among pregnant women in Munyenge, south west region, Cameroon following scale-up of communal piped water sources from 2014 to 2017: A crosssectional study. BMC Public Health. 2019;19(1):1-10.

18. Anderson RM, Truscott JE, Pullan RL, Brooker SJ, Hollingsworth TD. How effective is school-based deworming for the community-wide control of soil-transmitted helminths? PLoS Negl Trop Dis. 2013;7(2):e2027.

19. Anderson R, Truscott J, Hollingsworth TD. The coverage and frequency of mass drug administration required to eliminate persistent transmission of soil-transmitted helminths. Philos Trans R Soc B Biol Sci. 2014;369(1645):1-14.

20. Brooker SJ, Mwandawiro CS, Halliday KE, Njenga SM, Mcharo C, Gichuki PM, et al. Interrupting transmission of soiltransmitted helminths: A study protocol for cluster randomised trials evaluating alternative treatment strategies and delivery systems in Kenya. BMJ Open. 2015;5(10):1-12.

21. Hardwick RJ, Truscott JE, Oswald WE, Werkman M, Halliday KE, Pullan RL, et al. Individual adherence to mass drug administration in neglected tropical disease control: A probability model conditional on past behaviour. PLoS Negl Trop Dis. 2021;15(1):e0009112. https://doi.org/10.1371/ journal.pntd.0009112.

22. Pullan RL, Halliday KE, Oswald WE, Mcharo C, Beaumont E, Kepha S, et al. Effects, equity, and cost of school-based and community-wide treatment strategies for soil-transmitted helminths in Kenya: a cluster-randomised controlled trial. Lancet. 2019;393(10185):2039-50. https://doi.org/10. 1016/S0140-6736(18)32591-1.

23. Legge H, Kepha S, Prochazka M, Halliday K, Pullan R, Gwayi-Chore MC, et al. Implementer and recipient perspectives of community-wide mass drug administration for soil-transmitted helminths in Kwale county, Kenya. PLoS Negl Trop Dis. 2020;14(4):1-18. https://doi.org/10.1371/journ al.pntd.0008258.

24. Halliday KE, Oswald WE, McHaro C, Beaumont E, Gichuki PM, Kepha S, et al. Community-level epidemiology of soiltransmitted helminths in the context of school-based deworming: baseline results of a cluster randomised trial on the coast of Kenya. PLoS Negl Trop Dis. 2019;13(8):1-22. https://doi.org/10.1371/journal.pntd.0007427.

25. Oswald WE, Halliday KE, McHaro C, Witek-McManus S, Kepha S, Gichuki PM, et al. Domains of transmission and association of community, school, and household sanitation with soil-transmitted helminth infections among children in coastal Kenya. PLoS Negl Trop Dis. 2019;13(11):1-16. https://doi.org/10.1371/journal.pntd.0007488.

26. Kihara JH, Kutima HL, Ouma J, Churcher TS, Changoma JM, Mwalisetso MA, et al. Urogenital schistosomiasis in women of reproductive age and pregnant mothers in Kwale County, Kenya. J Helminthol. 2015;89:105-11.

27. WHO. World Malaria Report. Geneva; 2020. p. 106-11.

28. (DNMP) D of NMP, ICF. Kenya Malaria Indicator Survey 2020. 2020;(Nairobi Kenya and Rockville Maryland):DNMP and ICF.

29. Ng'etich A, Voyi K, Mutero C. Feasibility of implementing recommendations to improve neglected tropical diseases surveillance and response in Kenya : A modified Delphi study. BMC Health Serv Res. 2021;21:1034-51. 
30. Kimani BW, Mbugua AK, Kihara JH, Ng'ang'a M, Njomo DW. Safety, efficacy and acceptability of praziquantel in the treatment of Schistosoma haematobium in pre-school children of Kwale County, Kenya. PLoS Negl Trop Dis. 2018;12(10):1-12. https://doi.org/10.1371/journal.pntd.0006852.

31. Njaanake KH, Vennervald BJ, Simonsen PE, Madsen H, Mukoko DA, Kimani $\mathrm{G}$, et al. Schistosoma haematobium and soil-transmitted helminths in Tana Delta district of Kenya: infection and morbidity patterns in primary schoolchildren from two isolated villages. BMC Infect Dis. 2016;16(1):110. https://doi.org/10.1186/s12879-016-1387-4.

32. Njenga SM, Mutungi FM, Wamae CN, Mwanje MT, Njiru KK, Bockarie MJ. Once a year school-based deworming with praziquantel and albendazole combination may not be adequate for control of urogenital schistosomiasis and hookworm infection in Matuga District, Kwale County, Kenya. Parasit Vectors. 2014;7(1):1-9 Available from: Parasites \& Vectors.

33. Truscott JE, Ower AK, Werkman M, Halliday K, Oswald WE, Gichuki PM, et al. Heterogeneity in transmission parameters of hookworm infection within the baseline data from the TUMIKIA study in Kenya. Parasit Vectors. 2019:12(1):1-13. https://doi.org/10.1186/s13071-019-3686-2.

34. Rutagwera DG, Tylleskär T. Co-infection with malaria, hookworm and schistosomiasis among school children in Zambezi: a school-based rapid survey. Med J Zambia. 2012;39(4):18-23.

35. Pullan RL, Gitonga C, Mwandawiro C, Snow RW, Brooker SJ. Estimating the relative contribution of parasitic infections and nutrition for anaemia among school-aged children in Kenya: A subnational geostatistical analysis. BMJ Open. 2013;3(2):1-10.

36. Bustinduy AL, Sutherland LJ, Chang-Cojulun A, Malhotra I, DuVall AS, Fairley JK, et al. Age-stratified profiles of serum IL-6, IL-10, and TNF-a cytokines among Kenyan children with Schistosoma haematobium, plasmodium falciparum, and other chronic parasitic co-infections. Am J Trop Med Hyg. 2015;92(5):945-51.

37. Chadeka EA, Nagi S, Sunahara T, Cheruiyot NB, Bahati F, Ozeki Y, et al. Spatial distribution and risk factors of Schistosoma haematobium and hookworm infections among schoolchildren in Kwale, Kenya. PLoS Negl Trop Dis. 2017;11(9):1-17. https://doi.org/10.1371/journal.pntd.0005872.

38. Sumbele IUN, Otia OV, Bopda OSM, Ebai CB, Kimbi HK, Nkuo-Akenji T. Polyparasitism with Schistosoma haematobium, plasmodium and soiltransmitted helminths in school-aged children in Muyuka- Cameroon following implementation of control measures: a cross sectional study. Infect Dis Poverty. 2021;10(1):14-29.

39. Kinung'Hi SM, Mazigo HD, Dunne DW, Kepha S, Kaatano G, Kishamawe C, et al. Coinfection of intestinal schistosomiasis and malaria and association with haemoglobin levels and nutritional status in school children in Mara region, northwestern Tanzania: A cross-sectional exploratory study. BMC Res Notes. 2017;10(1):1-11.

40. Kwale County Government. Kwale County Nutrition Capacity assessment: Pilot Report. 2017. Available from: http://www.nutritionhealth.or.ke/wpcontent/uploads/Downloads/Kwale County Capacity Assessment Report - July 2017.pdf.

41. KNBS. 2019 Kenya Population and Housing Census Volume IV: Distribution of Population by Socio-Economic Characteristics 2019. Available from: https://www.knbs.or.ke/?wpdmpro=2019-kenya-population-andhousing-census-volume-iv-distribution-of-population-by-socio-econo mic-characteristics

42. Bisanzio D, Mutuku F, LaBeaud AD, Mungai PL, Muinde J, Busaidy H, et al. Use of prospective hospital surveillance data to define spatiotemporal heterogeneity of malaria risk in coastal Kenya. Malar J. 2015;14(1):482-93.

43. Cochran WG. Sampling technique. 2nd ed. Ney York: John Wiley and Sons Inc:; 1963.

44. ODK. ODK Documentation; 2020. p. i-678. Available from: https://docs. getodk.org

45. Clennon JA, Mungai PL, Muchiri EM, King CH, Kitron U. Spatial and temporal variations in local transmission of schistosoma haematobium in Msambweni, Kenya. Am J Trop Med Hyg. 2006;75(6):1034-41.

46. Amollo DA, Kihara JH, Kombe Y, Karanja SM. Prevalence and intensity of single and mixed Schistosoma Mansoni and Schistosoma Haematobium infections in primary school children in Rachuonyo North District, Homabay County, Western Kenya. East Afr Med J. 2013;90(2):1-9.

47. Montresor A, Crompton DWT, Hall A, Bundy DAP, Savioli L. Guidelines for the evaluation of soil-transmitted helminthiasis and schistosomiasis at community level. World Heal Organ WHO/CTD/SIP/981 1998,
48. Ojja S, Kisaka S, Ediau M, Tuhebwe D, Kisakye AN, Halage AA, et al. Prevalence, intensity and factors associated with soil-transmitted helminths infections among preschool-age children in Hoima district, rural western Uganda. BMC Infect Dis. 2018;18:408-19.

49. Ivoke N, Ikpor N, Ivoke O, Ekeh F, Ezenwaji N, Odo G, et al. Geophagy as risk behaviour for gastrointestinal nematode infections among pregnant women attending antenatal clinics in a humid tropical zone of Nigeria. Afr Health Sci. 2017;17(1):24-31.

50. Shinondo C, Mwikuma G. Geophagy as A risk factor for helminth infections in pregnant women in Lusaka, Zambia. Med J Zambia. 2009;35(2):48-52.

51. Jia TW, Melville S, Utzinger J, King CH, Zhou XN. Soil-transmitted helminth reinfection after drug treatment: A systematic review and meta-analysis. PLoS NegI Trop Dis. 2012;6(5):e1621-e1631.

52. Malhotra I, McKibben M, Mungai P, McKibben E, Wang X, Sutherland LJ, et al. Effect of antenatal parasitic infections on anti-vaccine lgG levels in children: A prospective birth cohort study in Kenya. PLoS Negl Trop Dis. 2015;9(1):1-18. https://doi.org/10.1371/journal.pntd.0003466.

53. Okoyo C, Campbell SJ, Williams K, Simiyu E, Owaga C, Mwandawiro C. Prevalence, intensity and associated risk factors of soil-transmitted helminth and schistosome infections in Kenya: impact assessment after five rounds of mass drug administration in Kenya. PLoS Negl Trop Dis. 2020;14(10):e0008604.

54. Mwandawiro C, Okoyo C, Kihara J, Simiyu E, Kepha S, Campbell SJ, et al. Results of a national school-based deworming programme on soil-transmitted helminths infections and schistosomiasis in Kenya: 20122017. Parasit Vectors. 2019;12:76-103.

55. Campbell SJ, Biritwum NK, Woods G, Velleman Y, Fleming F, Stothard JR. Tailoring water, sanitation, and hygiene (WASH) targets for soiltransmitted helminthiasis and schistosomiasis control. Trends Parasitol. 2018;34(1):1689-99. https://doi.org/10.1016/j.pt.2017.09.004.

56. Espinosa Aranzales AF, Radon K, Froeschl G, Pinzón Rondón ÁM, Delius M. Prevalence and risk factors for intestinal parasitic infections in pregnant women residing in three districts of Bogotá, Colombia. BMC Public Health. 2018;18(1):1-15.

57. Bisanzio D, Mutuku F, Bustinduy AL, Mungai PL, Muchiri EM, King CH, et al. Cross-sectional study of the burden of vector-borne and soil-transmitted Polyparasitism in rural communities of Coast Province, Kenya. PLoS Negl Trop Dis. 2014;8(7):e2992-3008.

58. Florey LS. Epidemiology of Polyparasitism in coastal Kenya: determinants, Interactions and Health Effects of Plasmodium Species and Schistosoma Haematobium Infections: University of Michigan; 2009.

59. Magak P, Chang-Cojulun A, Kadzo H, Ireri E, Muchiri E, Kitron U, et al. Case-control study of posttreatment regression of urinary tract morbidity among adults in schistosoma haematobium-endemic communities in kwale county, Kenya. Am J Trop Med Hyg. 2015;93(2):371-6.

60. Mutuku FM, King CH, Bustinduy AL, Mungai PL, Muchiri EM, Kitron U. Impact of drought on the spatial pattern of transmission of Schistosoma haematobium in coastal Kenya. Am J Trop Med Hyg. 2011;85(6):1065-70.

61. Poggensee G, Krantz I, Kiwelu I, Feldmeier H. Screening of Tanzanian women of childbearing age for urinary schistosomiasis: validity of urine reagent strip readings and self-reported symptoms. Bull World Health Organ. 2000;78(4):542-8.

62. Salawu OT, Odaibo AB. Schistosomiasis among pregnant women in rural communities in Nigeria. Int J Gynecol Obstet. 2013;122:1-4.

63. Eyo JE, Onyishi GC, Okafor FC. Urinary schistosomiasis among pregnant women in some endemic tropical semi - urban communities of Anambra state, Nigeria. Trop Biomed. 2012;29(4):575-9.

64. Rogerson SJ, Mwapasa V, Meshnick SR. Malaria in pregnancy: linking immunity and pathogenesis to prevention. Am J Trop Med Hyg. 2007;77(SUPPL. 6):14-22.

65. Cutts JC, Agius PA, Lin Z, Powell R, Moore K, Draper B, et al. Pregnancyspecific malarial immunity and risk of malaria in pregnancy and adverse birth outcomes : a systematic review. BMC Med. 2020;18:14-34.

66. Yang D, He Y, Wu B, Deng Y, Li M, Yang Q, et al. Drinking water and sanitation conditions are associated with the risk of malaria among children under five years old in sub-Saharan Africa : A logistic regression model analysis of national survey data. J Adv Res. 2020;21:1-13.

67. Jaleta KT, Hill SR, Birgersson G, Tekie H, Ignell R. Chicken volatiles repel host-seeking malaria mosquitoes. Malar J. 2016;15(1):354-62.

68. Florey LS, King CH, van Dyke MK, Muchiri EM, Mungai PL, Zimmerman PA, et al. Partnering parasites: evidence of synergism between heavy 
schistosoma haematobium and plasmodium species infections in Kenyan children. PLoS Negl Trop Dis. 2012;6(7):1-11.

69. Utzinger J, Rinaldi L, Lohourignon LK, Rohner F, Zimmermann MB,

Tschannen AB, et al. FLOTAC: a new sensitive technique for the diagnosis of hookworm infections in humans. Trans R Soc Trop Med Hyg. 2008;102(1):84-90.

70. Miller-Fellows SC, Howard L, Kramer R, Hildebrand V, Furin J, Mutuku FM, et al. Cross-sectional interview study of fertility, pregnancy, and urogenital schistosomiasis in coastal Kenya: documented treatment in childhood is associated with reduced odds of subfertility among adult women. PLoS Negl Trop Dis 2017;11(11):1-13. https://doi.org/10.1371/ journal.pntd.0006101

\section{Publisher's Note}

Springer Nature remains neutral with regard to jurisdictional claims in published maps and institutional affiliations.

- fast, convenient online submission

- thorough peer review by experienced researchers in your field

- rapid publication on acceptance

- support for research data, including large and complex data types

- gold Open Access which fosters wider collaboration and increased citations

- maximum visibility for your research: over $100 \mathrm{M}$ website views per year

At BMC, research is always in progress.

Learn more biomedcentral.com/submissions 